\title{
Boundary-layer phase transition in nematic liquid crystals
}

\author{
Ping Sheng \\ Conorate Research Science Laboratories, Exxon Research and Engineering Company, \\ P. O. Box 45, Linden, New Jersey 07036
}

(Received 1 March 1982)

\begin{abstract}
Phase-transition properties of nematic liquid crystals aligned by a short-range, arbitrarystrength-substrate potential are examined in the framework of Landau-de Gennes theory. It is shown that the substrate potential, which can arise from surface treatment of liquidcrystal display cells, not only induces a boundary layer in which the order-parameter values can be significantly different from that of the bulk, but also introduces a new "boundarylayer phase transition" which occurs at temperatures higher than the bulk-transition temperature. This novel transition is found to take place only in a limited range of substrate potential strength. For 4-pentyl-4'-cyanobiphenyl (PCB), the limiting values of this range are computed to be $\sim 0.075$ and $\sim 0.15 \mathrm{erg} / \mathrm{cm}^{2}$. Calculations are performed for both the semi-infinite-sample case and the finite-thickness-sample case. Various phase diagrams are presented to show the effects of sample thickness and substrate potential on the bulk as well as the boundary-layer phase-transition temperatures. The paper concludes with a discussion of experimental possibilities.
\end{abstract}

\section{INTRODUCTION}

Substrate alignment of nematic liquid crystals is a widely used technique to produce uniform or twisted director configurations in liquid-crystal display cells. In an earlier work, ${ }^{1}$ it was theoretically predicted that if the alignment of the nematic liquid crystals were anchored at the nematicsubstrate interface, then there is a "boundary layer", extending about $1000 \AA$ from the substrate, within which the degree of orientational ordering of the nematic liquid crystal could be significantly different from that of the bulk. It was further shown that if the liquid-crystal cell were comparable to or thinner than the boundary-layer thickness, then the first-order nematic-isotropic phase transition could disappear altogether. In the subsequent work ${ }^{2}$ by Miyano, the existence of the boundary layer was experimentally verified, although the difficulties in preparing nematic cells thinner than the boundary layer have prevented the confirmation of phasetransition modification effects. However, optical measurements of the boundary layer revealed some interesting details. For example, the experimental data are consistent with an order parameter value of

$$
S \equiv\left\langle P_{2}(\cos \theta)\right\rangle \simeq 0.3
$$

at the substrate-nematic interface, where $P_{2}$ denotes the second Legendre polynomial, $\theta$ is the angle between the long axis of a molecule and the director, and \langle\rangle means spatial averaging. Since the interface alignment of liquid crystals is due to the interaction between liquid-crystal molecules and the substrate, the relatively small value of $S$ implies that the strength of the substrate potential is much weaker than what a rigid anchoring condition would require. It follows that the previous calculation based on the anchoring condition is no longer adequate for a realistic description of the experimental system. It is the purpose of this note to examine the consequences of a short-range, arbitrarystrength-substrate potential. The results of a Landau - de Gennes theory calculation indicate that while the qualitative behavior of the boundary layer remains the same as in the previous work, there is, surprisingly, a new "boundary-layer phase transition" that occurs at a temperature separate from the bulk transition temperature $T_{K}^{0}$. This boundarylayer transition is found to take place only in a limited range of the substrate potential strength $G$, and can be observed either in bulk (semi-infinite) samples or in finite-thickness samples down to a thickness of $\sim 1000 \AA$ (at which point the boundarylayer transition merges with the bulk transition). In what ensues, the nematic-substrate interaction and the nature of the boundary-layer phase transition in a semi-infinite sample are examined in Sec. II. The phase-transition properties in a finite-thickness sample are calculated in Sec. III. In Secs. IV and V, we present the relevant phase diagrams and con- 
clude with a brief discussion of experimental possibilities.

\section{SEMI-INFINITE SAMPLE PROBLEM}

Consider a sample of nematic liquid crystal bounded on one side by a substrate. The solidliquid-crystal interface is defined as $z=0$, and the sample is assumed to be uniform in the $x$ and $y$ directions. The substrate is treated so that the nematic liquid-crystal molecules in its immediate vicinity experience an uniaxial aligning potential along some fixed spatial direction $\hat{n}$. The potential felt by each molecule can be expressed in general as $^{3}$

$$
\begin{aligned}
v(\theta, z)=-G \delta(z)[ & P_{2}(\cos \theta)+b P_{4}(\cos \theta) \\
& \left.+c P_{6}(\cos \theta) \cdots\right],
\end{aligned}
$$

where $\theta$ is the angle between the long axis of the molecule, $\hat{n}, z$ is the normal distance from the substrate, $G$ is a constant denoting the strength of the potential, $P_{2 n}$ denotes even-order Legendre polynomials, and $b, c$, etc., are the series expansion coefficients for the angular part of the potential. In Eq. (1), we have assumed the potential to be short range in nature, as evident from the delta function $\delta(z)$. If, in addition, $v(\theta, z)$ is truncated to the leading term of the series and averaged over the many molecules within a small spatial region, then the resulting form of the macroscopic potential is given by

$$
\begin{aligned}
V=\langle v(\theta, z)\rangle & =-G \delta(z)\left\langle P_{2}(\cos \theta)\right\rangle \\
& =-G \delta(z) S .
\end{aligned}
$$

To study the thermodynamic consequences of such a substrate potential, we start with the Landau-de Gennes free-energy density ${ }^{4} \phi$ :

$$
\begin{aligned}
& \phi=f(S)+L\left[\frac{d S}{d z}\right]^{2}-\frac{G}{A} \delta(z) S, \\
& f(S)=a\left(T-T^{*}\right) S^{2}+B S^{3}+C S^{4},
\end{aligned}
$$

where $T$ is the temperature, $a, T^{*}, B, C$, and $L$ are material parameters which can be determined from thermodynamic and fluctuation measurements, and $A$ is the area of the planar substrate. Given $\phi$, the total free energy $\Phi$ is obtained directly by integration over $z$ :

$$
\frac{\Phi}{A}=\int_{0}^{\infty}\left[f(S)+L\left[\frac{d S}{d z}\right]^{2}\right] d z-\frac{G}{A} S_{0},
$$

where $S_{0}$ denotes the value of $S$ at $z=0$. To determine the equilibrium form of $S(z)$, we employ the condition of minimum free energy. The minimization procedure involves two steps: First, $S_{0}$ is held fixed and the integral in Eq. (5) is minimized variationally with respect to $S(z)$. The resulting expression for $\Phi$ is then minimized with respect to $S_{0}$. Implementation of the first step results in the Euler equation

$$
f^{\prime}(S)=2 L\left(\frac{d^{2} S}{d z^{2}}\right),
$$

which can be integrated once to yield

$$
L\left(\frac{d S}{d z}\right)^{2}=f(S)+K .
$$

The constant $K$ is determined by the condition that at $z \rightarrow \infty$, the bulk liquid crystal is uniform and, therefore,

$$
\left.\frac{d S}{d z}\right|_{z \rightarrow \infty}=0
$$

which directly implies

$$
\xi_{0}^{2}\left(\frac{d S}{d z}\right)^{2}=F(S)-F\left(S_{b}\right) .
$$

Here $\xi_{0} \equiv\left(L / a T_{K}^{0}\right)^{1 / 2}$ is the correlation length, $F(S) \equiv f(S) / a T_{K}^{0}$, and $S_{b}$ is the bulk value of the order parameter. Substitution of Eq. (8) into Eq. (5) yields

$$
\begin{aligned}
\frac{\Phi}{A a T_{K}^{0}}= & F\left(S_{b}\right) D \\
& +\xi_{0}\left[2 \int_{S_{b}}^{S_{0}} \sqrt{F(S)-F\left(S_{b}\right)} d S-g S_{0}\right]
\end{aligned}
$$

where $D$ is the sample thickness $\left(D / \xi_{0} \rightarrow \infty\right.$ in the present case), and $g \equiv G / A \xi_{0} a T_{K}^{0}$ is the dimensionless parameter characterizing the substrate potential. In Eq. (9), the expression for $\Phi$ clearly consists of two parts. The first term, the bulk free-energy part, is proportional to $D$. The second term, the boundary-layer part $\Phi_{\mathrm{BL}}$, can be picked out by its proportionality to $\xi_{0}$ :

$$
\frac{\Phi_{\mathrm{BL}}}{A \xi_{0} a T_{K}^{0}}=2 \int_{S_{b}}^{S_{0}} \sqrt{F(S)-F\left(S_{b}\right)} d S-g S_{0} .
$$

The equilibrium value of $S_{0}$ is determined by the condition $d \Phi_{\mathrm{BL}} / d S_{0}=0$, or

$$
F\left(S_{0}\right)=F\left(S_{b}\right)+\frac{g^{2}}{4}
$$


where $S_{b}$ is found by the stipulation that

$$
F\left(S_{b}\right)=\min F(S),
$$

where min represents the absolute minimum of $F(S)$. In case Eq. (11) has multiple roots, the correct $S_{0}$ is that one which gives the lowest value of $\Phi_{\mathrm{BL}}$ as expressed by Eq. (10). Once $S_{0}$ is found, the $S(z)$ profile can be obtained through the integral of Eq. (8), or

$$
\frac{z}{\xi_{0}}=\int_{S(z)}^{S_{0}} \frac{d S}{\sqrt{F(S)-F\left(S_{b}\right)}}
$$

In Fig. 1 we show the calculated result of $S_{0}$ as a function of temperature for the nematic liquidcrystal 4-pentyl-4'-cyanobiphenyl (PCB). In the calculation, measured values ${ }^{5} a=0.065 \mathrm{~J} / \mathrm{cm}^{3} \mathrm{~K}$, $B=-0.53 \mathrm{~J} / \mathrm{cm}^{3}, C=0.98 \mathrm{~J} / \mathrm{cm}^{3}, T^{*}=307.14 \mathrm{~K}$, and $L=4.5 \times 10^{-14} \mathrm{~J} / \mathrm{cm}$ were used. ${ }^{6}$ It is seen that for $g<0.0056, S_{0}$ experiences a discontinuous transition in its value at the bulk-transition temperature $T_{K}^{0}$. However, at

$$
0.0056=g_{0}<g<g_{c}=0.012
$$

the transition of $S_{0}$ occurs at a temperature higher than $T_{K}^{0}$. At $g>g_{c}$, the transition disappears and the variation of $S_{0}$ becomes a continuous function of temperature. The nature of the transition at $g>g_{c}$ and in the range $g_{0}<g<g_{c}$ deserves closer scrutiny. In Fig. 2, we show the behavior of the boundary layer for $g=0.004$. At $T=T_{K}^{0}$, it is seen that both $S_{0}$ and $S_{b}$ experience a discontinuous jump as indicated by the two curves corresponding to $S(z)$ just before and just after the transition. The simultaneous transition of $S_{0}$ and $S_{b}$ is understandable since the value of $S_{0}$ is linked to the bulk $S_{b}$ by elastic forces [represented by the $L(d S / d z)^{2}$ term in the free-energy density] and in the limit of weak substrate potential the transition in $S_{0}$ is induced by the bulk transition. At $g=0.008>g_{0}$, however, a different type of boundary-layer behavior is predicted as illustrated in Fig. 3. Figure 3(a) shows the $S(z)$ just before and just after the bulk transition at $T_{K}^{0}$. Although $S_{b}$ has a discontinuous jump, $S_{0}$ stays fixed. At $T=T_{K}^{0}+0.049 \mathrm{~K}$, on the other hand, the value of $S_{0}$ exhibits a discontinuous transition while $S_{b}$ stays unchanged $(=0)$ as illustrated in Fig. 3(b). Since, in this case, the first-order transition involves only the boundary layer, we call the phase change shown in Fig. 3(b) the boundary-layer phase transition. The physics of this occurrence is

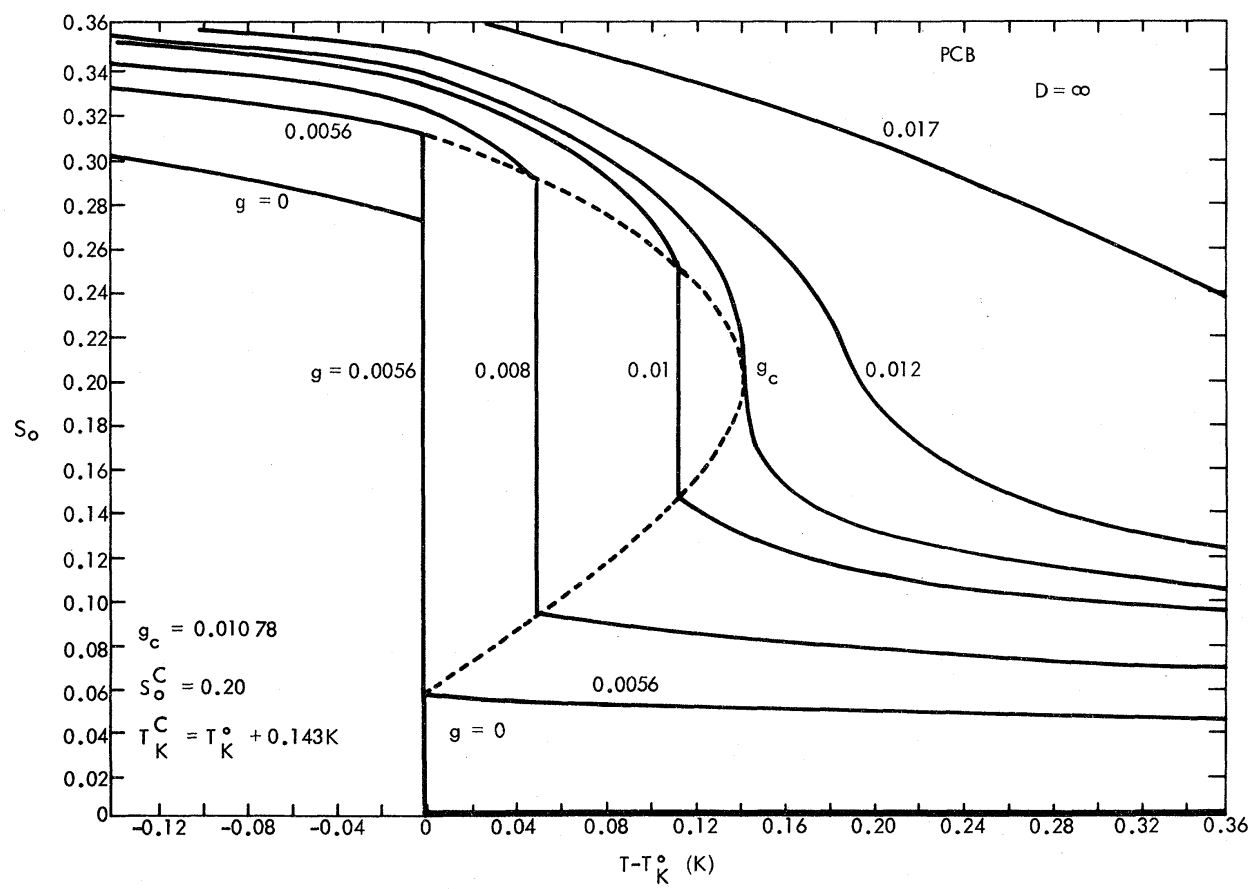

FIG. 1. Calculated PCB order-parameter value at the substrate-nematic interface plotted as a function of temperature. Magnitude of the substrate potential is labeled beside each curve. Values of the critical point parameters are noted in the figure. 


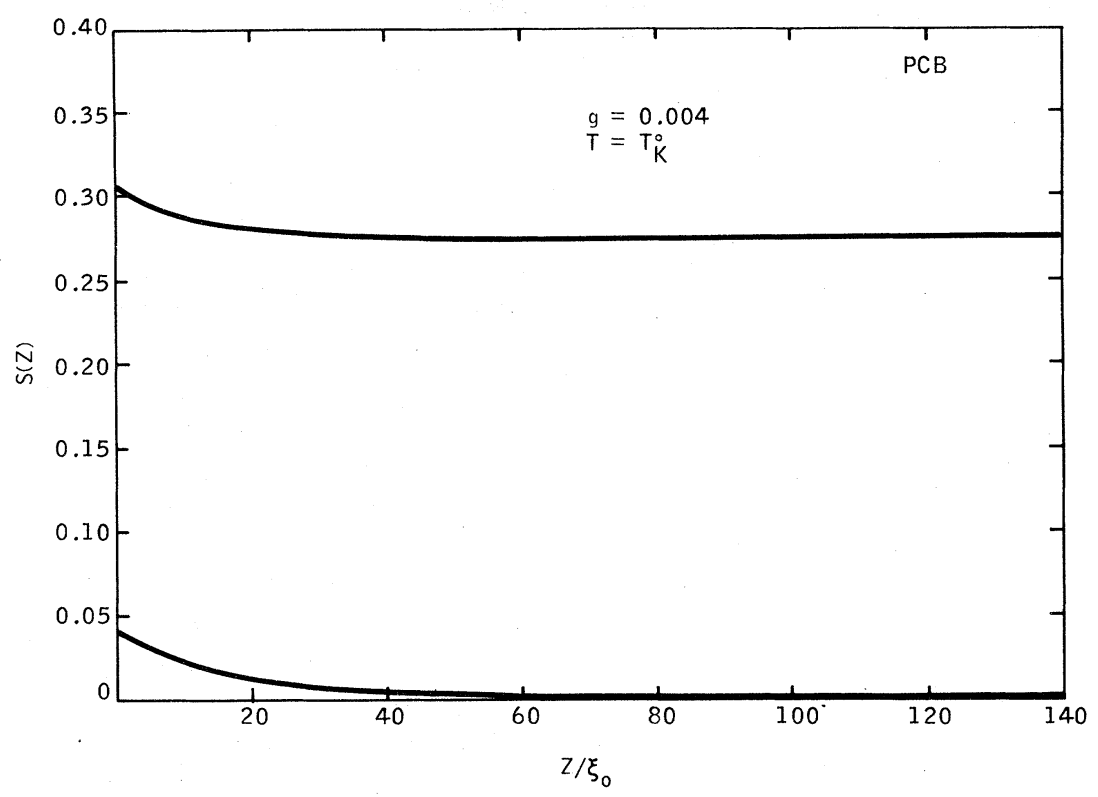

FIG. 2. Variation of the order parameter $S$ as a function of distance $z / \xi_{0}$ away from the substrate. Upper curve shows $S(z)$ just before the bulk transition at $T=T_{K}^{0}$, and the lower curve shows $S(z)$ just after the transition. Value of the substrate potential $g=0.004$ is noted in the figure.

actually fairly simple. The layer of nematic molecules at the liquid-crystal-substrate interface experiences two forces: the elastic force, which connects the surface molecules with the bulk, and the substrate aligning force. When the substrate potential is sufficiently strong, i.e., $g>g_{0}$ (but $g<g_{c}$ ), the increase in the elastic part of the free energy caused by the lowering of the bulk order-parameter value at $T_{K}^{0}$ cannot overcome the surface aligning potential. Therefore, $S_{0}$ stays unchanged. However, as temperature increases beyond $T_{K}^{0}$, there is a point at which a trade off between the elastic free energy and the surface potential energy becomes advantageous and a boundary-layer transition occurs in which the gain in surface potential energy (resulting from the decrease of $S_{0}$ ) is offset by the lowering of the elastic free energy (and vice versa when $T$ is lowered through the transition temperature).

The existence of an upper limiting value of $g$ for the boundary-layer phase transition can also be made plausible. From the form of the substrate potential $V=-G \delta(z) S$, it is clear that we can regard the interface alignment as due to the application of a localized magnetic field ${ }^{7} H$, with $G \propto H^{2}$. Since it is well known that the nematic-isotropic transition has a critical point under a strong magnetic field, ${ }^{8}$ it follows by analogy that the first-order transition in $S_{0}$ should also possess a critical point $\left(T_{K}^{c}, g_{c}\right)$ at which the first-order boundary-layer phase transition becomes second order. For PCB, the value of $g_{0}=0.0056$ and $g_{c}=0.012$ translate into a substrate potential $G / A \xi_{0}$ of roughly 0.15 and $0.3 \mathrm{~J} / \mathrm{cm}^{3}$, respectively. Since $\xi_{0} \equiv \sqrt{L / a T_{K}^{0}} \simeq 5 \AA$, this means

$$
G_{0} / A \simeq 0.075 \mathrm{erg} / \mathrm{cm}^{2}
$$

and

$$
G_{c} / A \simeq 0.15 \mathrm{erg} / \mathrm{cm}^{2} .
$$

\section{FINITE-THICKNESS SAMPLE PROBLEM}

Consider a sample of nematic liquid crystal of uniform thickness $2 D$ sandwiched between two identically treated substrates situated at $z=0$ and $z=2 D$. Instead of the condition $d S / d z(z=\infty)=0$ for the semi-infinite sample, in this case we have $d S / d z(z=D)=0$ due to the symmetry of the problem. Therefore, if we let $S_{b}$ denote the orderparameter value at middle of the sample, i.e., $z=D$, then we are led to exactly Eq. (9), where $\Phi$ now stands for half of the total free energy of the sample. However, due to the fact that $D$ is finite, $S_{b}$ and $S_{0}$ can no longer be decoupled as in the previous case. Therefore, we have to solve the coupled equations

$$
F\left(S_{0}\right)-F\left(S_{b}\right)=\frac{g^{2}}{4},
$$




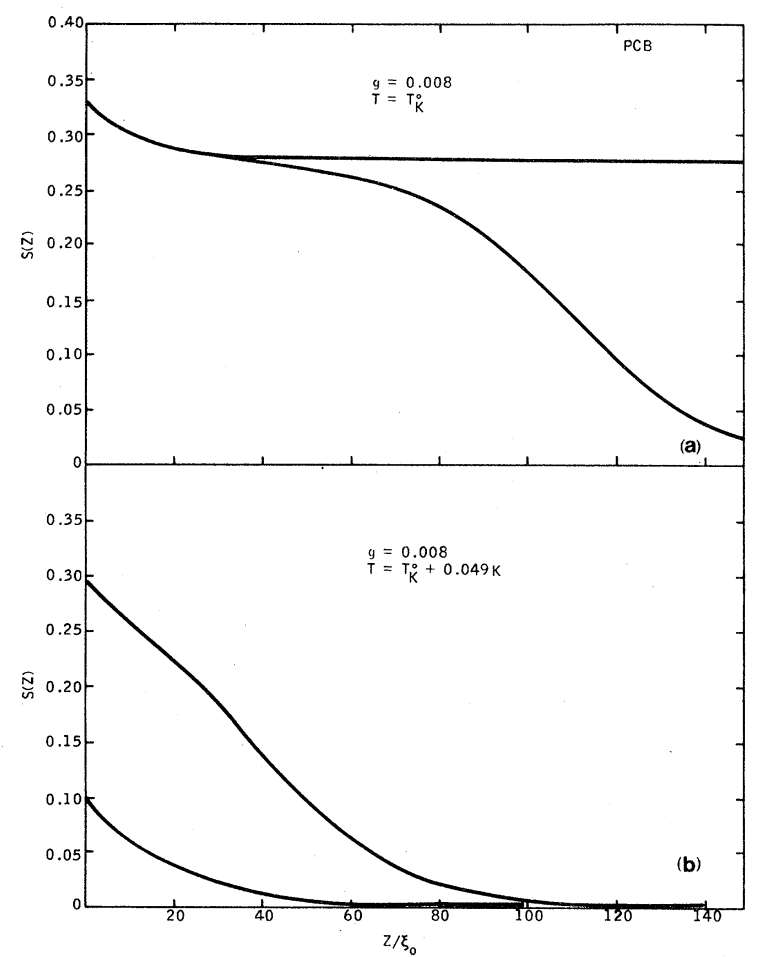

FIG. 3. (a) Variation of the order parameter $S$ as a function of distance $z / \xi_{0}$ away from the substrate. Two curves show $S(z)$ just before and just after the bulk transition at $T=T_{K}^{0}$. Magnitude of the substrate potential $g=0.008$ is noted in the figure. In this case, the bulk order parameter has a discontinuous jump, but the value of $S_{0}$ stays fixed. (b) Variation of $S$ as a function of $z / \xi_{0}$ at $T=T_{K}^{0}+0.049 K$. Substrate potential is the same as in (a). Two curves correspond to $S(z)$ just before and just after a transition has occurred. Since in this case the transition involves only the boundary layer, it is labeled the boundary-layer phase transition.

$$
F^{\prime}\left(S_{b}\right)\left(1-\frac{\xi_{0}}{D} \int_{S_{b}}^{S_{0}} \frac{d S}{\sqrt{F(S)-F\left(S_{b}\right)}}\right)=0
$$

When there are multiple pairs of solutions $\left(S_{0}, S_{b}\right)$, the correct pair is always that one which gives the lowest value of $\Phi$ as expressed by Eq. (9). It should be noted that when $\xi_{0} / D \rightarrow 0$, Eq. (13) reduces to Eq. (11) for the semi-infinite case.

Through the use of the measured parameter values of $\mathrm{PCB}$, we have calculated the variations of $S_{b}$ and $S_{0}$ as a function of $T, g$, and $D$. For $g=0.008$, it is seen from Fig. 4 that the transition temperature of $S_{b}$ increases with decreasing $D$. As to $S_{0}$, at $D / \xi_{0}=300$, it shows two transitions: the boundary-layer phase transition plus the one induced by the transition of $S_{b}$. We will label the transition which causes discontinuities in both $S_{0}$ and $S_{b}$ as the "bulk transition," in accordance with its limiting characterization as $D / \xi_{0} \rightarrow \infty$. Since the boundary-layer transition temperature is found to be invariant with respect to $D$, the increasing bulk-transition temperature means that there is a thickness $D / \xi_{0}=160$, below which the boundarylayer transition disappears into the bulk transition. As $D$ decreases even further, the first-order transitions in both $S_{0}$ and $S_{b}$ eventually turn into second-order transitions at $D / \xi_{0}=15$. Results of similar calculations with $g=0.012>g_{c}$ are plotted in Fig. 5. In contrast to the semi-infinite case in which $S_{0}$ has no abrupt transition at this value of the substrate potential, for finite sample thickness $S_{0}$ always experiences a discontinuous jump coincident with the first-order transition in $S_{b}$. This

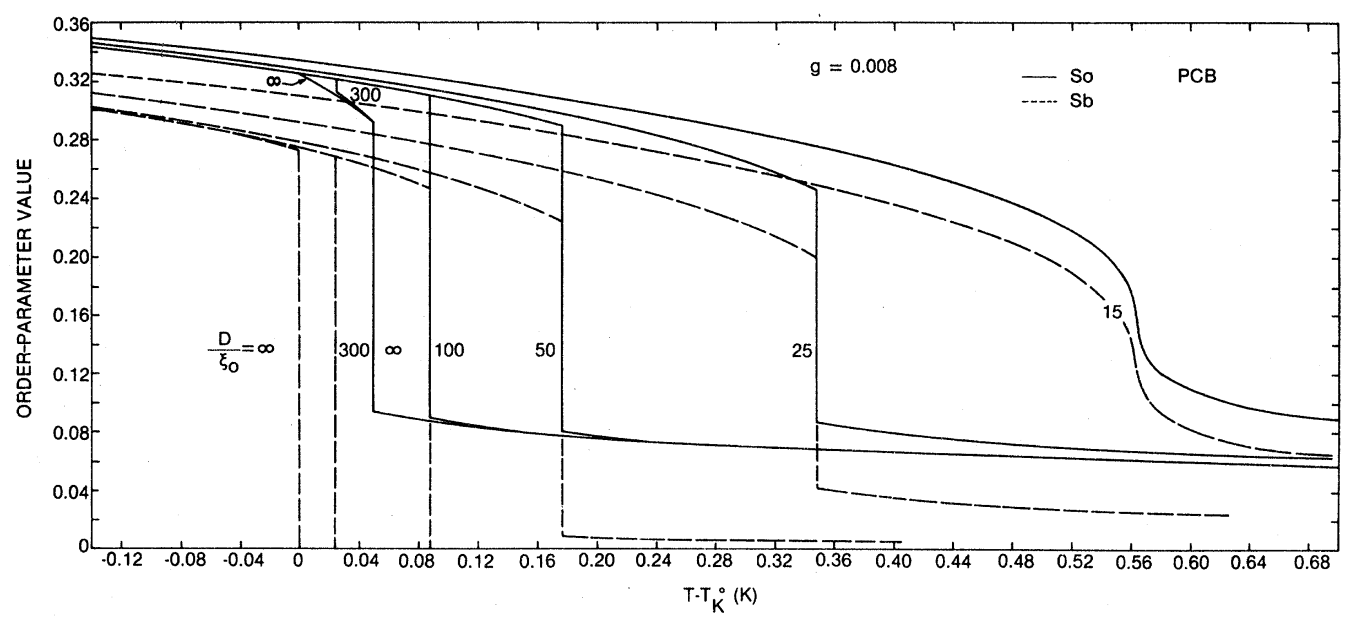

FIG. 4. Variation of $S_{b}$ (dashed curve) and $S_{0}$ (solid curve) as a function of temperature. The (half) thickness of the sample is labeled beside each curve. Magnitude of the substrate potential, $g=0.008$, is noted in the figure. 


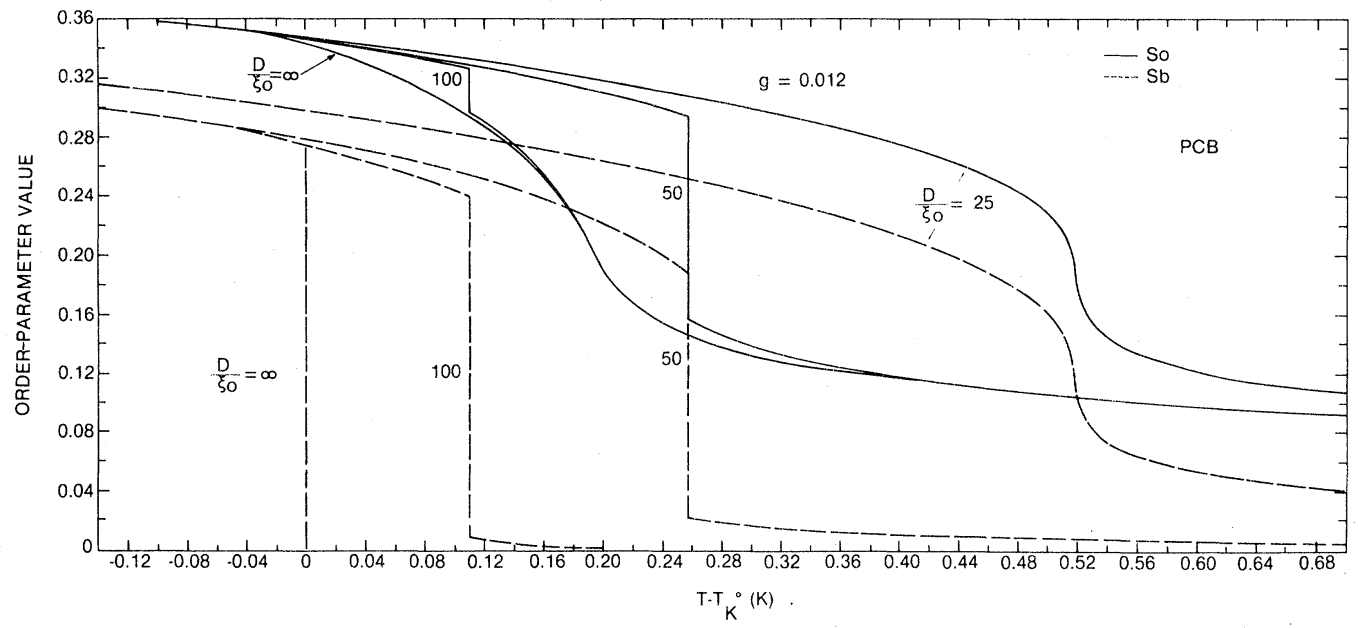

FIG. 5. Variation of $S_{b}$ (dashed curve) and $S_{0}$ (solid curve) as a function of temperature. The (half) thickness of the sample is labeled beside each curve. Magnitude of the substrate potential, $g=0.012$, is noted in the figure.

behavior is similar to that for $g=0.008$ at $D / \xi_{0}<160$. The critical thickness in this case occurs at $D / \xi_{0} \simeq 25$.

\section{PHASE DIAGRAMS}

Figures 6 and 7 show the thickness-temperature phase diagrams for sandwiched PCB samples of thickness $2 D$. Since in finite-thickness samples the liquid-crystal molecules exhibit a certain degree of surface-induced orientational ordering at all temperatures, we have labeled the high-temperature phase "paranematic" in analogy to the paramagnetic phase in magnetism. In Fig. 6, the solid line delineates the variation of bulk first-order phasetransition temperature as a function of $D / \xi_{0}$ for $g=0.008$. The boundary-layer transition is marked by the dot-dashed line. It is noted that the most dramatic increase in the bulk-transition temperature

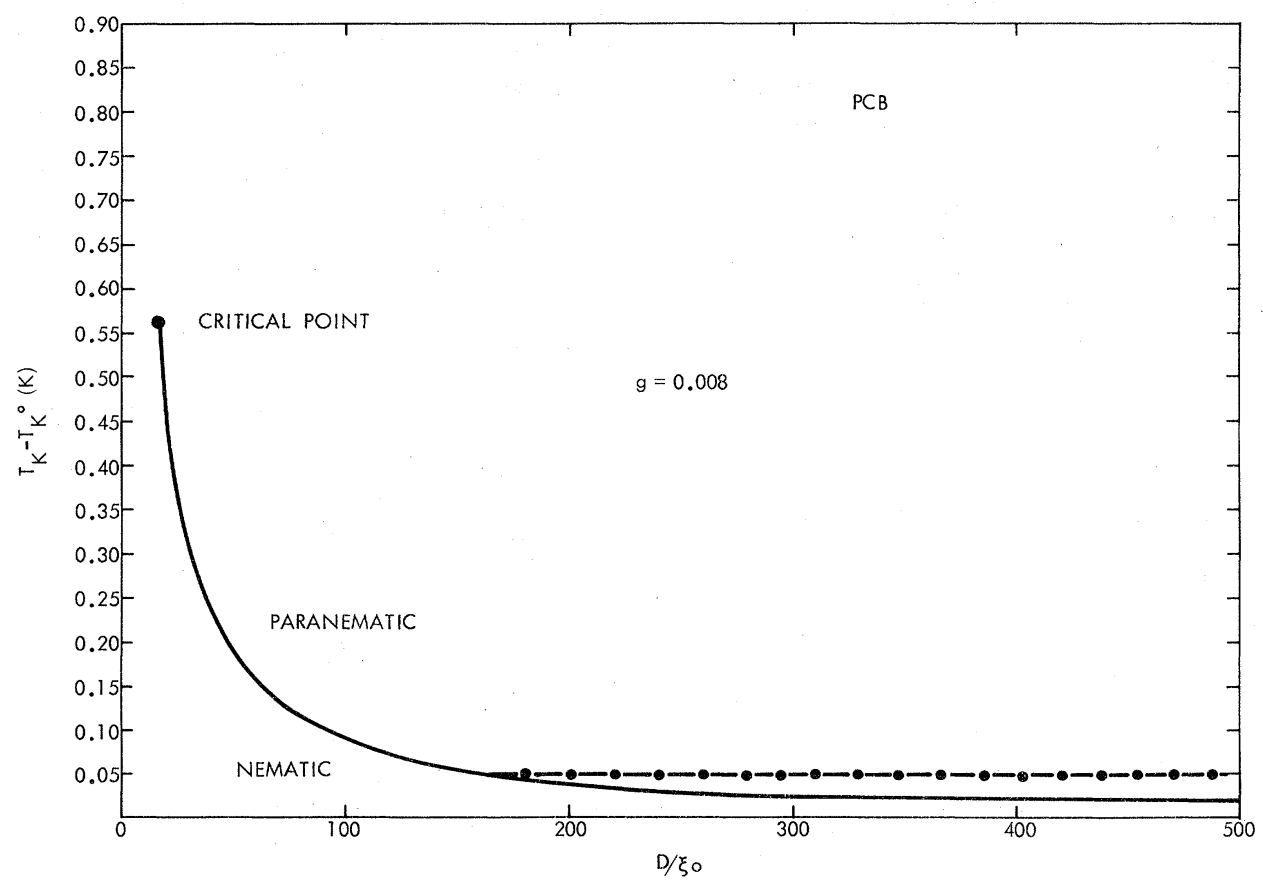

FIG. 6. Temperature-thickness phase diagram at $g=0.008$. Boundary-layer transition is denoted by the dot-dashed line. 


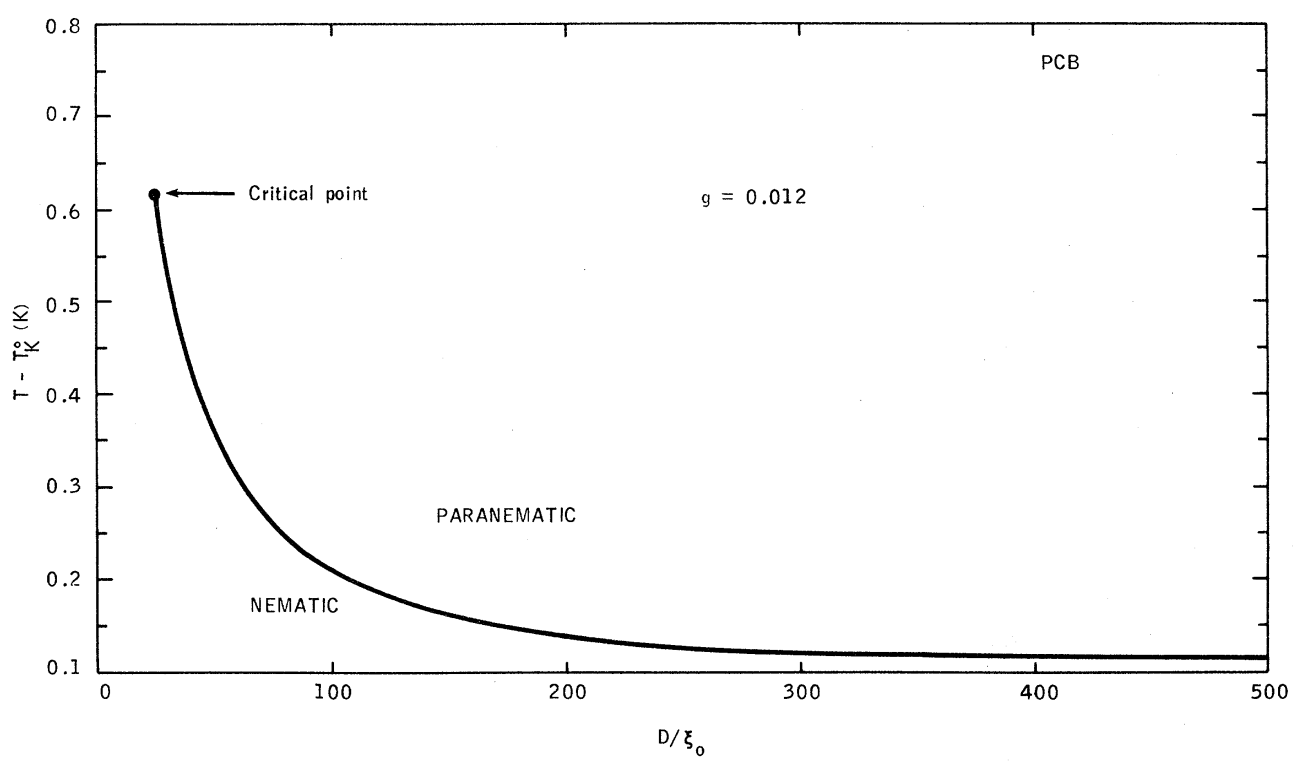

FIG. 7. Temperature-thickness phase diagram at $g=0.012$.

occurs at $D / \xi_{0}<100$. Figure 7 is the similar phase diagram for $g=0.012$. Since at this value of the substrate potential there is no longer a boundarylayer phase transition, only the bulk-transition temperature is shown. In Fig. 8 the phase-transition temperatures are plotted as a function of $g$. It should be noted that whereas the bulk-transition temperatures (solid curves) are labeled by their respective sample thicknesses, the boundary-layer transition temperature, denoted by the dot-dashed line, requires no such labeling since it remains the same for all sample thicknesses. Yet the point of appearance for the boundary-layer phase transition, marked by the intersection of the solid and the dotdashed lines, is thickness dependent. This is due to the fact that the boundary-layer phase transition only occurs at temperatures higher than the bulktransition temperature.

\section{DISCUSSION}

Many aspects of the theoretical predictions that we have discussed above are open to experimental verification. In this section we will discuss the following three possibilities:

(1) Measurement of $S_{0}(T)$. At present the temperature dependence for the integral $\int S(z) d z$ has been measured, and the experimental data were interpreted by assuming ${ }^{2}$ a fixed $S_{0}$ (a reanalysis of

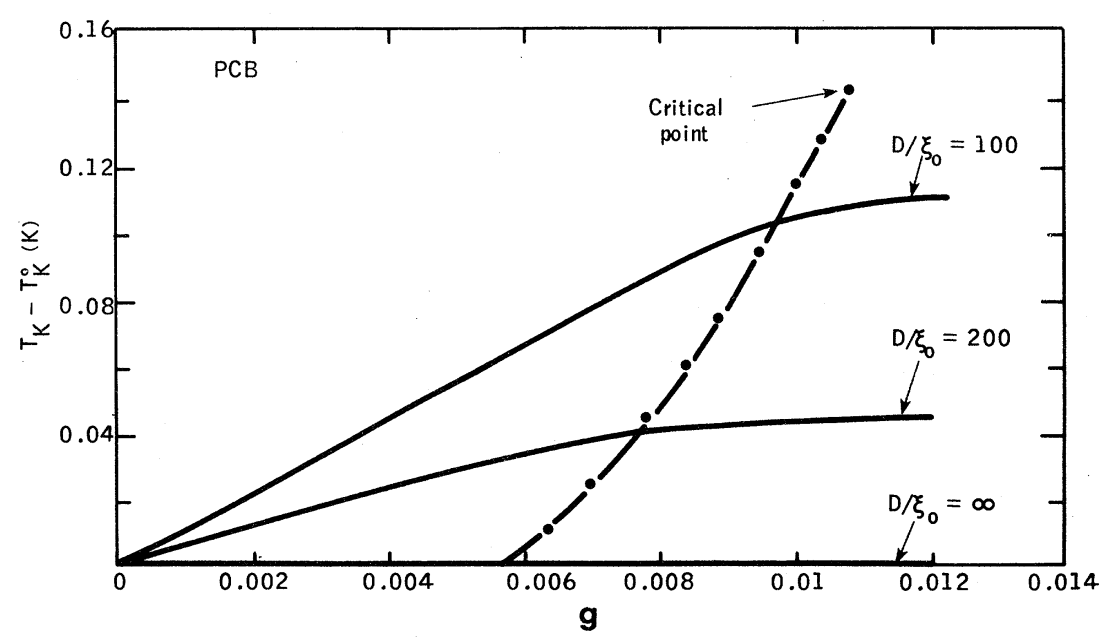

FIG. 8. Bulk (solid line) and the boundary-layer (dot-dashed line) phase-transition temperatures plotted as a function of the substrate potential strength $g$. The (half) thickness of the samples are labeled beside each solid curve. 
the data in terms of the present theory will be published elsewhere ${ }^{9}$ ). However, since the anchoring of $S_{0}$ would imply an infinite-strength substrate potential, it seems reasonable that $S_{0}$ should vary in any real system, and a measurement of that variation would provide a direct determination of the substrate potential strength $G$. Probably a more refined interferometric method along the line of Mada and Kobayashi's experiment ${ }^{2}$ would be a feasible technique for such measurement.

(2) Direct observation of the boundary-layer phase transition. Potentially the most difficult part of such an experiment is the control of surface treatment so that the resulting substrate potential lies in the range $g_{0}<g<g_{c}$. One possible approach is to generate, on a single substrate, a continuous set of surface conditions so that the correct range of substrate potential is present at part of the sample. An additional benefit of this technique, if achievable, is the possible observation of the critical point $\left(T_{K}^{c}, g_{c}\right)$.

(3) Verification of the finite-thickness effect. Since the most significant departure from bulksample behavior occurs at $D / \xi_{0}<100$, sample thicknesses of $2000 \AA$ and below are required. If the thickness can be made variable in a single experimental run (such as by using a wedge-type sample), then one can measure the critical behavior when the first-order phase transition becomes second order. Besides providing further insights into cooperative effects in thin films, such measurements would undoubtedly pose new challenges for more refined theoretical calculations.

In closing, it should be remarked that the predictions of this paper are based on the assumption that the Landau - de Gennes theory is valid in the neighborhood of the nematic-isotropic phase transition. Although the experimental evidences so far offer strong support for the Landau-de Gennes model (on its prediction of the pretransitional behaviors) it is generally agreed that the order-parameter expansion or the mean-field approach cannot yield correct theoretical behaviors close to a critical point. In this context, the theoretical predictions presented in this paper can also be viewed as a test of the Landau-de Gennes theory. A negative experimental result on any of the predictions therefore would be interesting since it can offer clues as to the direction of further theoretical refinements.

\section{ACKNOWLEDGMENT}

The author wishes to acknowledge many helpful discussions with Professor Y. R. Shen.
${ }^{1}$ Ping Sheng, Phys. Rev. Lett. 37, 1059 (1976).

${ }^{2}$ K. Miyano, J. Chem. Phys. 71, 4108 (1979); see also Phys. Rev. Lett. $\underline{43}$, 51 (1979). In a separate measurement, H. Mada and S. Kobayashi [Appl. Phys. Lett. $\underline{35}, 4(1979)]$ have obtained semiquantitative results on the value of $S_{0}$.

${ }^{3}$ Angular dependence of any uniaxial potential can always be expanded in a series involving only the evenorder Legendre functions. See, for example, P. Sheng and P. J. Wojtowicz, Phys. Rev. A 14, 1883 (1976).

${ }^{4}$ P. G. de Gennes, Mol. Cryst. Liq. Cryst. 12, 193 (1971). In Eq. (3) the substrate potential is added to the origi- nal form of the Landau-de Gennes free-energy density.

${ }^{5}$ H. J. Coles, Mol. Cryst. Liq. Cryst. 49, 67 (1978).

${ }^{6}$ Estimated value from the measurement on MBBA.

${ }^{7}$ See, for example, Introduction to Liquid Crystals, edited by E. B. Priestley, P. J. Wojtowicz, and P. Sheng (Plenum, New York, 1975), p. 162.

8P. J. Wojtowicz and P. Sheng, Phys. Lett. 48A, 235 (1974).

${ }^{9} \mathrm{P}$. Sheng, in Proceedings of the Fourth International Symposium on Liquid Crystals and Ordered Fluids, Las Vegas, Nevada, March, 1982 (in press). 